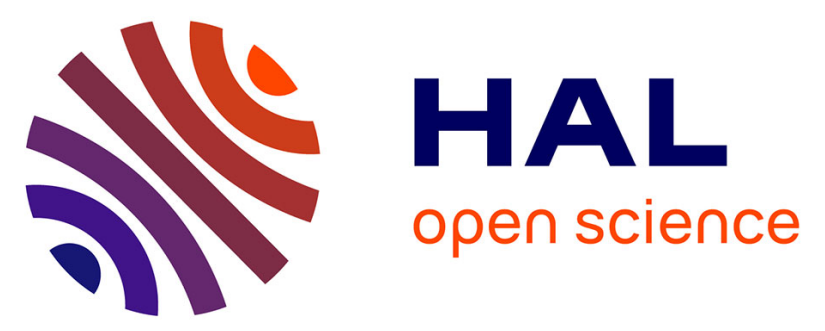

\title{
À propos du comportement de butinage de Megachile sculpturalis Smith, 1853, en France méditerranéenne (Nîmes et Montpellier) (Hymenoptera, Megachilidae)
}

Valérie Andrieu-Ponel, Philippe Ponel, Violette Le Féon, Benoit Geslin, Gérard Duvallet

\section{To cite this version:}

Valérie Andrieu-Ponel, Philippe Ponel, Violette Le Féon, Benoit Geslin, Gérard Duvallet. À propos du comportement de butinage de Megachile sculpturalis Smith, 1853, en France méditerranéenne (Nîmes et Montpellier) (Hymenoptera, Megachilidae). Bulletin de la Société Entomologique de France, 2018, 123 (1), pp.49-54. hal-01742472

HAL Id: hal-01742472

https://hal.science/hal-01742472

Submitted on 27 Mar 2018

HAL is a multi-disciplinary open access archive for the deposit and dissemination of scientific research documents, whether they are published or not. The documents may come from teaching and research institutions in France or abroad, or from public or private research centers.
L'archive ouverte pluridisciplinaire HAL, est destinée au dépôt et à la diffusion de documents scientifiques de niveau recherche, publiés ou non, émanant des établissements d'enseignement et de recherche français ou étrangers, des laboratoires publics ou privés. 


\title{
À propos du comportement de butinage de Megachile sculpturalis Smith, 1853, en France méditerranéenne (Nîmes et Montpellier) (Hymenoptera, Megachilidae)
}

\author{
Valérie Andrieu-Ponel ${ }^{1}$, Philippe Ponel ${ }^{1}$, Violette Le FéON ${ }^{2}$, \\ Benoît GesLiN ${ }^{3}$ \& Gérard Duvallet ${ }^{4}$ \\ ${ }^{1}$ Aix Marseille Univ, Univ Avignon, CNRS, IRD, IMBE, Marseille, France, \\ Technopôle Arbois-Méditerranée, bât. Villemin, BP 80, F - 13545 Aix-en- Provence cedex 04 \\ $<$ valerie.andrieu-ponel@imbe.fr><philippe.ponel@imbe.fr> \\ ${ }^{2}$ Observatoire des Abeilles, 2 domaine Bellevue, F - 11290 Arzens <violettelf@hotmail.com> \\ ${ }^{3}$ Aix Marseille Univ, Univ Avignon, CNRS, IRD, IMBE, Marseille, France, site Saint-Jérôme, \\ avenue de l'Escadrille Normandie-Niémen, F-13013 Marseille < benoit.geslin@imbe.fr> \\ ${ }^{4}$ Centre d'Écologie fonctionnelle et évolutive, UMR5175, Université Paul-Valéry Montpellier, route de Mende, \\ F - 34199 Montpellier cedex 5 <gduvallet@aol.com>
}

(Accepté le 11.I.2018)

Résumé. - L'étude du contenu pollinique de deux échantillons prélevés, soit sur un spécimen de Megachile sculpturalis Smith, 1853, soit dans la loge d'un "hôtel à insectes" fréquenté par cet Hyménoptère, livre des indications préliminaires sur le comportement de butinage et sur le régime alimentaire de cette abeille invasive dont la première observation en France date de 2008. La domination des assemblages polliniques par le pollen de Sophora, une Fabaceae arborescente originaire d'Asie et plantée essentiellement dans les parcs et jardins en France, témoigne de la préférence de Megachile sculpturalis pour cette essence, au moins dans la localité étudiée. L'étude se basant seulement sur deux échantillons, de nouvelles analyses sont nécessaires pour approfondir les connaissances sur ses besoins alimentaires et pour statuer sur une éventuelle préférence ou spécialisation sur Sophora japonica pour la collecte du pollen.

Abstract. - About the foraging behavior of Megachile sculpturalis Smith, 1853, in Mediterranean France (Nîmes and Montpellier) (Hymenoptera, Megachilidae). The study of the pollen content of two samples taken, either on one specimen of Megachile sculpturalis Smith, 1853, or in the lodge of an "insect hotel" frequented by this Hymenoptera, gives preliminary indications on the behaviour of foraging and on the diet of this invasive bee whose first observation in France dates back to 2008. The dominance of pollen assemblages by the pollen of Sophora, an arborescent Fabaceae native to Asia and planted mainly in parks and gardens in France, testifies to the preference of Megachile sculpturalis for this species, at least in the studied area. Further investigations are needed to know to what extent M. sculpturalis prefers collecting pollen on Sophora japonica.

Keywords. - Invasive bee, diet, Sophora, pollen.

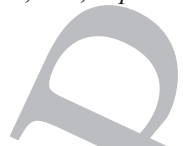

Megachile sculpturalis Smith, 1853, est une Abeille solitaire et xylicole, originaire d'Asie orientale (VEREECKEN \& BARBIER, 2009). La dissémination hors de son aire d'origine de cette espèce ou d'autres espèces de la famille de Megachilidae semble être associée au commerce du bois, et c'est à partir des grandes zones portuaires que se ferait la migration de cette abeille. Aux États-Unis, M. sculpturalis a été observée pour la première fois en Caroline du Nord, en 1994 (Mangum \& Brooks, 1997). En France, c'est en juillet 2008 que les premiers individus ont été repérés, sur la commune d'Allauch, près du port de Marseille (VEREECKEN \& BARBIER, 2009). Depuis, l'espèce s'est largement répandue en France et en Europe (Allemagne, Autriche, Italie et Suisse). En France, l'espèce a été détectée dans 72 communes entre 2008 et 2016. Elle est bien présente dans le quart sud-est et a progressé en 2016 jusqu'à Mâcon au nord, et jusqu'au Pays Basque à l'ouest (Le FÉon et al., 2018). 
L'objectif de cette note est de compléter les connaissances sur le régime alimentaire de cette Abeille en France. Plusieurs auteurs ont mis en évidence que Megachile sculpturalis se nourrissait de nombreuses espèces de plantes (États-Unis : MANGum \& SuMNER, 2003; HinojosaDiAz et al., 2005 ; PARYs et al., 2015 ; Italie : QuARANTA et al., 2012 en Italie; France : LE FÉON et al., 2018). Cependant, les études comprenant une analyse du pollen fourni aux larves par les femelles sont rares. Les analyses palynologiques sont indispensables pour qualifier une abeille de polylectique (i.e. la femelle nourrit ses larves de pollen pouvant provenir de plantes de différentes familles) ou oligolectique (i.e. le pollen est collecté sur des plantes de la même famille), voire monolectique (i.e. le pollen est collecté sur une seule espèce de plante). En Italie, QUARANTA et al. (2012) ont trouvé que le contenu d'une cellule larvaire était majoritairement composé du genre Ligustrum. Dans le sud de la France, Westrich et al. (2015) indiquent que le contenu de cellules larvaires issues d'une localité située à $30 \mathrm{~km}$ au nord de Manosque (département des Alpes-de-Haute-Provence) contenait majoritairement du pollen de Sophora japonica. Cette espèce, de la famille des Fabaceae, est originaire d'Asie et a été introduite en France (Muséum national d'Histoire naturelle, à Paris) en 1747 (REEB, 2010). C'est un arbre mellifère, dont le pollen et le nectar sont particulièrement appréciés par les abeilles. Dans le sud de la France, cet arbre fleurit en été et à l'automne. On le retrouve assez fréquemment planté le long des routes, dans les parcs urbains ou dans les jardins des particuliers. Le degré de préférence de M. sculpturalis pour Sophora japonica en France n'est pas encore clairement établi. Nous présentons ici les résultats d'analyses palynologiques permettant d'améliorer les connaissances. Cependant, l'étude que nous présentons est préliminaire car elle repose sur l'analyse pollinique d'un nombre faible d'échantillons.

\section{MATÉRIEL ET MÉTHODE}

Les échantillons. - Deux échantillons polliniques ont été étudiés. L'échantillon $\mathrm{n}^{\circ} 1$ provient d'un jardin privé de Nîmes (Gard, France). Il a été récupéré au pinceau par M. R. Jeantet (ancien directeur du Muséum d'Histoire naturelle de Nîmes) sur l'abdomen de plusieurs individus de Megachile sculpturalis qui revenaient dans leurs loges d'un "hôtel à insectes". Le prélèvement a été fait début juillet 2015. L'échantillon ${ }^{\circ} 2$ a été prélevé dans une loge d'un hôtel à insectes du jardin de SupAgro de Montpellier (Hérault, France) le 1 $1^{\text {er }}$ juillet 2015, juste après l'émergence d'un adulte de Megachile sculpturalis.

Technique d'extraction du pollen et calcul des pourcentages. - Les échantillons ont été déshydratés avec de l'acide acétique puis, après rinçage à l'alcool à l'aide d'une centrifugeuse, le culot a été acétolysé, rincé à l'alcool et à l'eau, mélangé à de la glycérine et monté entre lame et lamelle. L'acétolyse est une hydrolyse à froid qui a la propriété de vider la cellule pollinique de son contenu cytoplasmique. C'est un mélange oxydant composé d'acide sulfurique $\left(1 / 9^{\mathrm{e}}\right)$ et d'anhydride acétique $\left(8 / 9^{\mathrm{e}}\right)$. À l'issue de ce traitement, le pollen est transparent, il est légèrement coloré de jaune par les produits utilisés et il est prêt à être observé au microscope photonique. Pour cette étude, une goutte de $25 \mu \mathrm{l}$ de culot par échantillon a été analysée. Les pourcentages de l'échantillon n 2 ont été calculés avec et sans Sophora afin de mieux faire apparaître les taxons dominants autres que Sophora (tableau I).

Morphologie du pollen de Sophora. - Sophora L. est un genre appartenant à la famille de Fabacées. Comme beaucoup de taxons de cette famille, le pollen de Sophora est tricolporé (PEÑA et al., 1993). Il est doté de longs sillons. En vue équatoriale, le grain est allongé et sphéroïdal. En vue polaire, il est circulaire. Son exine est finement réticulée (fovéolée). La taille des grains de Sophora observés en vue équatoriale est de $20 \mu \mathrm{m}$ (fig. 1). 
Bulletin de la Société entomologique de France, 12X (X), 201X : 000-000

Tableau I. - Comptage pollinique de l'échantillon 2 (Montpellier, Hérault, France) (t. : type pollinique).

\begin{tabular}{|c|c|c|c|}
\hline Taxa & Valeurs brutes & \% avec Sophora & \% sans Sophora \\
\hline Anthemis t. & 20 & 0,24 & 7,22 \\
\hline Aster t. & 42 & 0,50 & 15,16 \\
\hline Betula sp. & 2 & 0,02 & 0,72 \\
\hline Cedrus sp. & 10 & 0,12 & 3,61 \\
\hline Centaurea nigra $\mathrm{t}$. & 1 & 0,01 & 0,36 \\
\hline Cerealia $40 \mu \mathrm{m}$ & 1 & 0,01 & 0,36 \\
\hline Chenopodiaceae & 1 & 0,01 & 0,36 \\
\hline Cichorioideae & 12 & 0,14 & 4,33 \\
\hline Cyperaceae & 3 & 0,04 & 1,08 \\
\hline Delitschia sp. & 3 & 0,04 & 1,08 \\
\hline Draba t. & 3 & 0,04 & 1,08 \\
\hline Geranium sp. & 1 & 0,01 & 0,36 \\
\hline Indéterminés & 2 & 0,02 & 0,72 \\
\hline Juniperus sp. & 5 & 0,06 & 1,81 \\
\hline Ligustrum sp. & 7 & 0,08 & 2,53 \\
\hline Mentha sp. & 3 & 0,04 & 1,08 \\
\hline Mycrothyrium sp. & 3 & 0,04 & 1,08 \\
\hline Olea sp. & 2 & 0,02 & 0,72 \\
\hline Picea sp. & 1 & 0,01 & 0,36 \\
\hline Pinus méditerranéens & 44 & 0,52 & 15,88 \\
\hline Pinus sylvestris t. & 1 & 0,01 & 0,36 \\
\hline Pistacia sp. & 1 & 0,01 & 0,36 \\
\hline Plantago coronopus t. & 10 & 0,12 & 3,61 \\
\hline Plantago lanceolata $\mathrm{t}$. & 9 & 0,11 & 3,25 \\
\hline Platanus sp. & 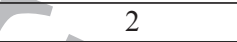 & 0,02 & 0,72 \\
\hline Poaceae & 2 & 0,02 & 0,72 \\
\hline Quercus caducifolié & 22 & 0,26 & 7,94 \\
\hline Quercus sempervirent & 28 & 0,33 & 10,11 \\
\hline Scabiosa sp. & 3 & 0,04 & 1,08 \\
\hline Sequoia sp. & 10 & 0,12 & 3,61 \\
\hline Sophora sp. & 8170 & 96,72 & / \\
\hline Spores de champignons indifférenciées & 16 & 0,19 & 5,78 \\
\hline Trifolium sp. & 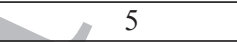 & 0,06 & 1,81 \\
\hline Viburnum sp. & 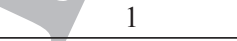 & 0,01 & 0,36 \\
\hline Vitis sp. & 7 & 0,01 & 0,36 \\
\hline Somme avec Sophora & 8447 & & \\
\hline Somme sans Sophora & 277 & & \\
\hline
\end{tabular}

\section{RÉSULTATS}

Le contenu pollinique de l'échantillon ${ }^{\circ} 1$ est exclusivement composé du pollen de Sophora sp. (fig. 1 et tableau I). Dans l'échantillon n², 8447 pollens ont été comptés dont 8170 grains de Sophora et 277 grains de pollen divers (tableau I). L'assemblage pollinique de l'échantillon $\mathrm{n}^{\circ} 2$ est plus diversifié mais il est très majoritairement dominé (ca $97 \%$ du total pollinique) par le pollen de Sophora sp.. Parmi les autres taxons identifiés, on observe la présence, dans l'ordre décroissant d'abondance, de pins méditerranéens, de chênes (caducifoliés et sclérophylles), d'Asteroideae (Aster type, Anthemis type, des Cichorioideae), de Plantago coronopus L., de Cedrus, de Sequoia et de champignons identifiés au niveau générique (Mycrothyrium sp. et la spore du champignon coprophile Delitschia sp.) ou pas (fig. 2). Parmi les Asteroideae, les grains 


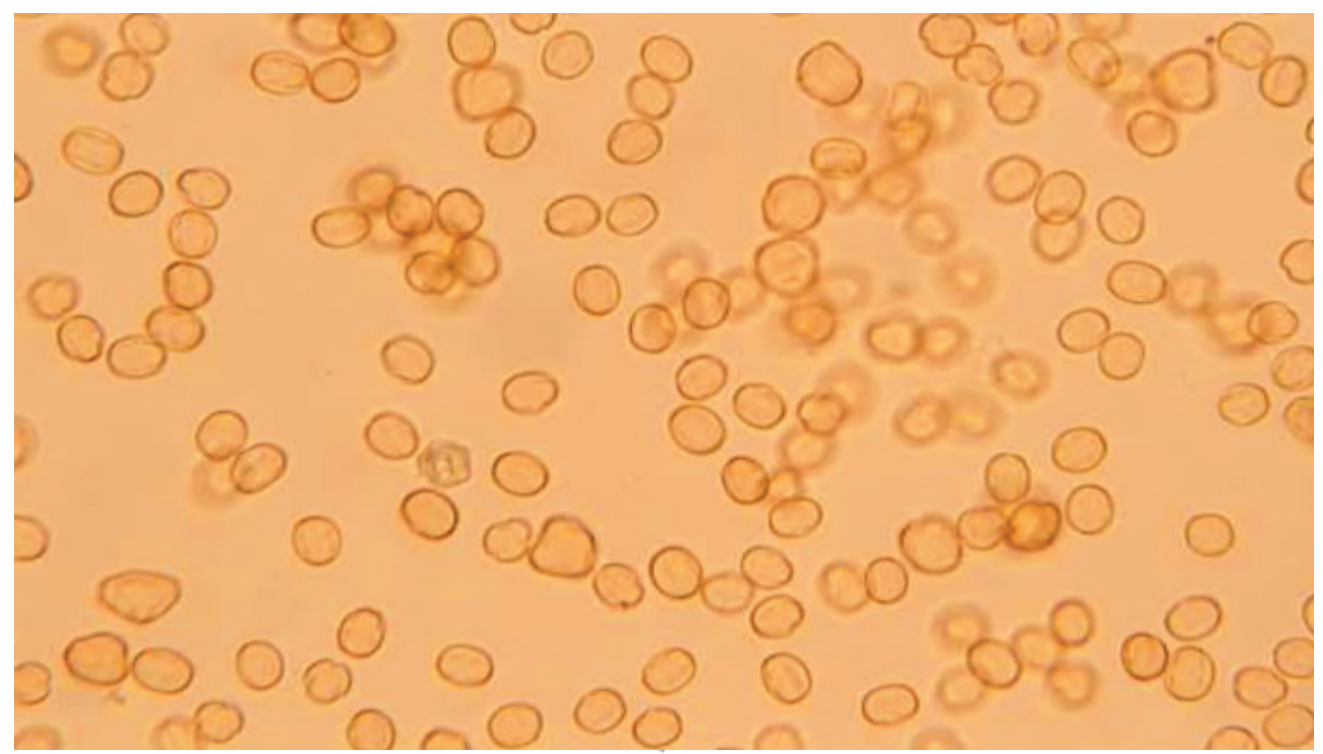

Fig. 1. - Assemblage pollinique de l'échantillon 1 (Nîmes, Gard, France). Noter que l'assemblage est exclusivement composé de pollen de Sophora sp. (longueur maximale d'un grain de pollen : $20 \mu \mathrm{m}$ ).

de pollen identifiés sous le type pollinique Aster ou Anthemis sont très similaires morphologiquement et semblent correspondre à une même espèce de plante (non identifiée) (fig. 2).

\section{Discussion}

La prépondérance du pollen de Sophora dans la charge pollinique transportée par Megachile sculpturalis (échantillon $\mathrm{n}^{\circ} 1$ ) montre clairement que, dans un cas au moins, cette espèce visite exclusivement les fleurs de cet arbre pour s'en nourrir et alimenter sa progéniture.

L'échantillon $n^{\circ} 2$, plus diversifié mais très largement dominé par le pollen de Sophora, pourrait correspondre à un mélange de pollen, celui transporté par $M$. sculpturalis et le pollen relictuel laissé dans les loges de l'hôtel à insectes par les occupants précédents. La présence de seulement deux espèces de plantes au sein de la sous-famille des Asteroideae qui comporte de nombreuses espèces mellifères, pourrait indiquer que l'abeille se nourrit aussi de ces plantes. Pour préciser les résultats sur le régime alimentaire de l'espèce, il faudrait effectuer l'analyse pollinique de nouveaux échantillons, travailler sur un nombre d'échantillons statistiquement représentatif, et associer ce travail à un relevé de végétation de la zone fréquentée par $M$. sculpturalis.

\section{Conclusion}

Les premiers résultats sont très prometteurs pour les futures études à mener sur la problématique de l'impact sur les populations d'insectes indigènes des espèces d'insectes exotiques récemment introduites. Concernant Megachile sculpturalis, il serait intéressant d'effectuer l'étude de son régime alimentaire en étudiant le contenu pollinique de son estomac, des pelotes de pollen qu'elle transporte et des cavités où elle nidifie, de préférence des loges d'hôtel à insectes neufs, pour éviter les pollutions. Afin de pouvoir tirer des conclusions solides, ces études devraient être conduites en France, en particulier près de la zone de première détection de cette abeille (Marseille), mais aussi à l'étranger, dans les régions où cette espèce a été introduite - Amérique du Nord (Mangum \& Brooks, 1997) — et dans les régions d'Asie d'où cette espèce est originaire. 


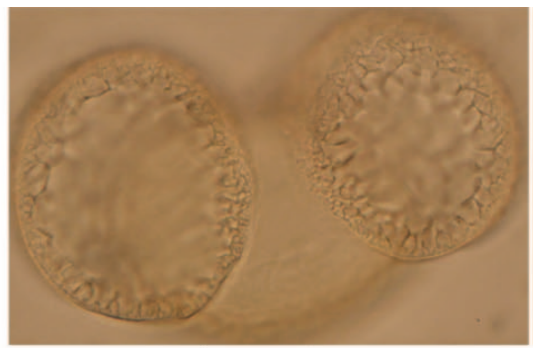

Pinus (méditerranéen), $\mathrm{L}=110 \mu \mathrm{m}$

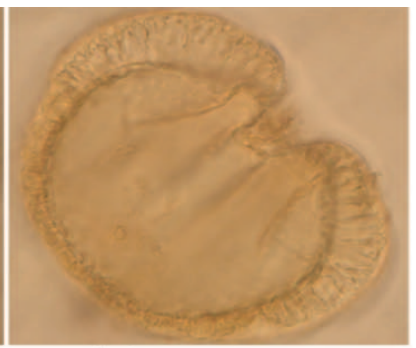

Cedrus sp., $\mathrm{L}=80 \mu \mathrm{m}$
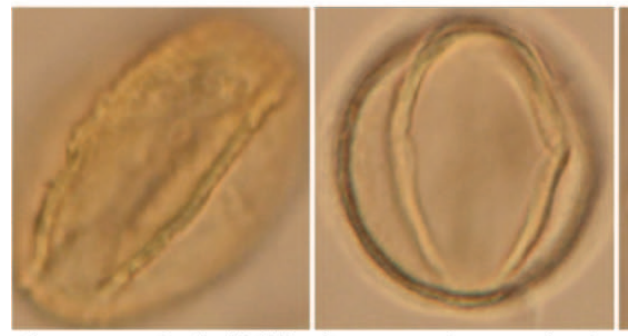

Quercus sp. (caducifolié), Quercus sp. (sempervirent), $\mathrm{L}=35 \mu \mathrm{m}$

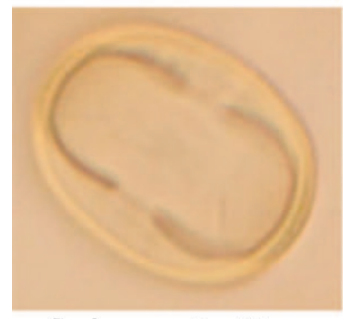

Sophora sp., $\mathrm{L}=20 \mu \mathrm{m}$

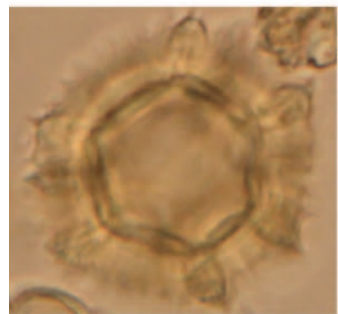

Cichorioideae, $\mathrm{L}=30 \mu \mathrm{m}$

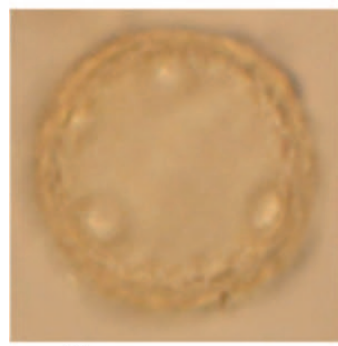

Plantago coronopus, $\mathrm{L}=25 \mu \mathrm{m}$

$$
\mathrm{L}=27,5 \mu \mathrm{m}
$$

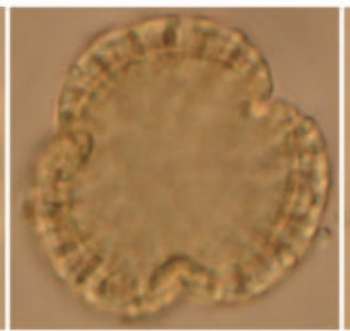

Ligustrum sp., $\mathrm{L}=30 \mu \mathrm{m}$

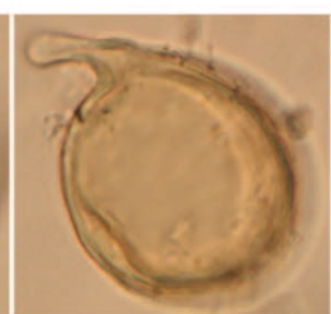

Sequoia sp., $\mathrm{L}=30 \mu \mathrm{m}$
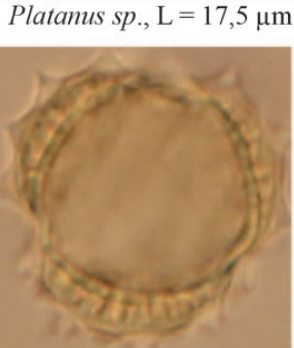

Anthemis t., $\mathrm{L}=27 \mu \mathrm{m}$

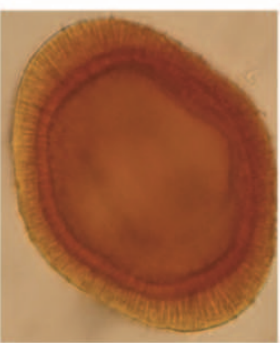

Scabiosa sp., $\mathrm{L}=75 \mu \mathrm{m}$

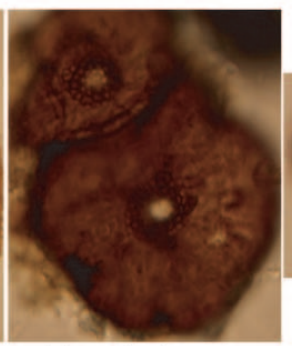

Mycrothyrium sp.,

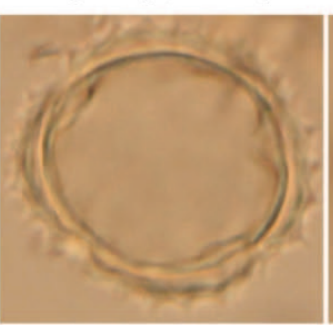

Aster t., $\mathrm{L}=25 \mu \mathrm{m}$

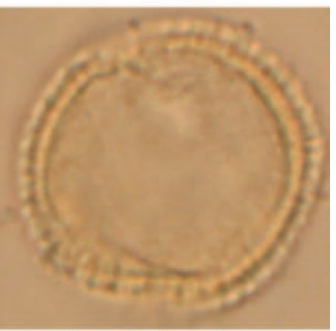

Olea sp., $\mathrm{L}=35 \mu \mathrm{m}$

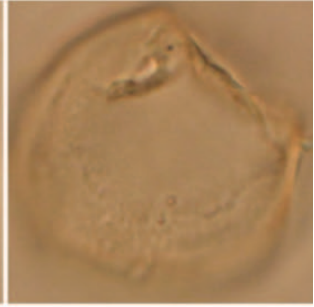

Poaceae, $\mathrm{L}=20 \mu \mathrm{m}$

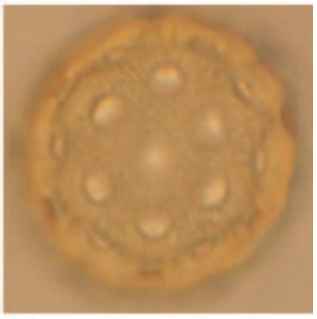

Chenopodiaceae, $\mathrm{L}=20 \mu \mathrm{m}$ $\mathrm{L}=70 \mu \mathrm{m}$

Fig. 2. - Pollens et spores de l'échantillon 2 (Montpellier, Hérault, France) $(\mathrm{L}=$ longueur maximale d'un grain de pollen ou d'une spore). 
ANDRIEu-Ponel et al. - Comportement de butinage de Megachile sculpturalis

RemerCiements. - Merci à René Jeantet de nous avoir procuré l'échantillon du Muséum de Nîmes, et à Davhya Belkacem d'avoir assuré le traitement des échantillons polliniques.

\section{Auteurs Cités}

Hinojosa-Diaz I. A., Yañez-Ordoñez O., Chen G., Peterson A. T. \& Engel M. S., 2005. - The North American invasion of the giant resin bee (Hymenoptera: Megachilidae). Journal of Hymenoptera Research, $14: 69-77$.

LAPORT R. G. \& MinCKLey R. L., 2012. - Occupation of active Xylocopa virginica nests by the recently invasive Megachile sculpturalis in upstate New York. Journal of the Kansas Entomological Society, 85 (4) : 384-386.

Le Féon V., Aubert M., Genoud D., Andrieu-Ponel V., Westrich P. \& Geslin B., 2018. - Range expansion of Megachile sculpturalis (Hymenoptera, Apoidea, Megachilidae) in France. Ecology \& Evolution. doi : 10.1002/ece3.3758.

Mangum W. A. \& BRooks R. W., 1997. - First records of Megachile (Callomegachile) sculpturalis Smith (Hymenoptera: Megachilidae) in the continental United States. Journal of the Kansas Entomological Society, 70 : 140-142.

Mangum W. A. \& SumNer S., 2003. - A survey of the North American range of Megachile (Callomegachile) sculpturalis, an adventive species in North America. Journal of the Kansas Entomological Society, 76 (4) : 658-662.

Parys K., Tripodi A. \& Sampson B., 2015. - The Giant Resin Bee, Megachile sculpturalis Smith: new distributional records for the Mid- and Gulf-south USA. Biodiversity Data Journal, 3 : e6733.

Peña R. C., Iturriaga L., Mujica A. M. \& Montenegro G., 1993. - Análisis micromorfológico de polen de Sophora (Papilionaceae). Hipótesis filogenética sobre el origen de la Sección Edwardsia. Gayana (Botánica), 50 (2) : 57-65.

Quaranta M., Sommaruga A., Balzarini P. \& Felicioli A., 2014. - A new species for the bee fauna of Italy: Megachile sculpturalis continues its colonization of Europe. Bulletin of Insectology, 67: 287-293.

ReeB C., 2010. - Plantes mellifères. Le Sophora du Japon. Abeilles \& Fleurs, 721 : 21-22.

VEREECKEN N. J. \& BARBIER E., 2009. - Premières données sur la présence de l'abeille asiatique Megachile (Callomegachile) sculpturalis Smith (Hymenoptera, Megachilidae) en Europe. Osmia, 3 : 4-6.

Westrich P., Knapp A. \& Berney I., 2015. - Megachile sculpturalis Smith 1853 (Hymenoptera, Apidae), a new species for the bee fauna of Germany, now north of the Alps. Eucera, 9:3-10.

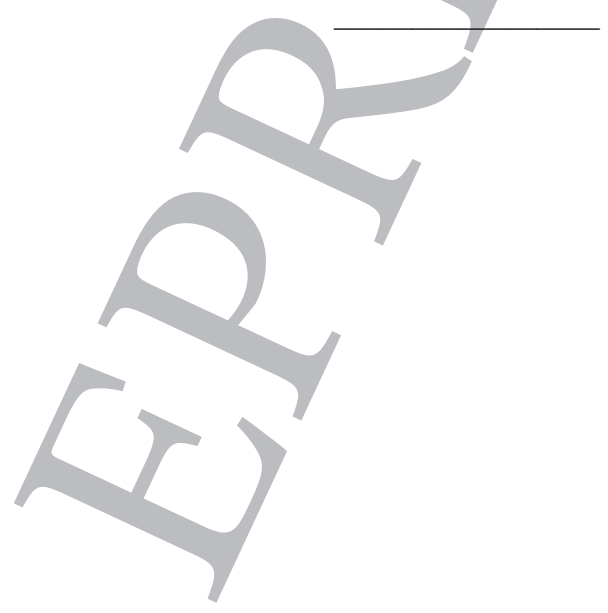

